\title{
Estimation of the Number of Cumulative COVID-19 Cases by Day in Thailand Based on a Flattened Curve Policy
}

\author{
Yupaporn AREEPONG ${ }^{1}$ and Rapin SUNTHORNWAT ${ }^{2, *}$ \\ ${ }^{I}$ Department of Applied Statistics, Faculty of Applied Science, King Mongkut's University of Technology \\ North Bangkok, Bangkok 10800, Thailand \\ ${ }^{2}$ Industrial Technology Program, Faculty of Science and Technology, Pathumwan Institute of \\ Technology, Bangkok 10330, Thailand
}

('Corresponding author's e-mail: rapin@pit.ac.th)

Received: 30 April 2020, Revised: 4 April 2021, Accepted: 11 April 2021

\begin{abstract}
Since December 2019, the world has been facing an emerging infectious disease named coronavirus disease 2019. Thailand has also been affected by the spread of the coronavirus. The Thai government have announced policies to protect people, based on the emergency decree and curfew law for flattening the curve of the number of the coronavirus disease 2019 cases without vaccination in Thailand. This research estimated of the number of total infectious cases of coronavirus disease 2019 in Thailand. Two growth curves, including an exponential growth curve under a non-flattened curve policy (herd immunity policy without vaccination), and a logistic growth curve under a flattened curve policy without vaccination, were selected to estimate the parameters of the curves by the least square method to represent the number of the total infectious cases in Thailand. Moreover, the maximum infectious cases of coronavirus disease 2019 and the speed of spreading for coronavirus disease 2019 in Thailand were also explored. Based on the number of the total infectious cases of coronavirus disease 2019 in Thailand, the findings demonstrated that the coefficient of determination of the logistic growth curve was greater than the exponential growth curve and the root means squared percentage error of the logistic growth curve was less than the exponential growth curve. These results suggest that the logistic growth curve is suitable for describing the number of total infectious cases of coronavirus disease 2019 in Thailand under the fattened curve policy.
\end{abstract}

Keywords: Coronavirus disease 2019, Estimating derivative, Exponential growth curve, Least square method, Logistic growth curve

\section{Introduction}

Coronavirus disease 2019 (COVID-19) is an emerging infectious disease caused by severe acute respiratory (pneumonia) and spread firstly in December 2019 at Wuhan, Hubei, China. Later, it has become a pandemic disease and widespread around the world. It can spread from person to person via touching the blood, body fluids, secretions, excretions of coronavirus disease 2019 patients. It severely spreads in Europe such as Spain, Italy, France, etc. as well as the United States of America. The first infectious case in Thailand was identified on January $13^{\text {th }}, 2020$. Thailand has abided by the guidance of the World Health Organization (WHO) related to protecting and controlling the disease outbreak in the world. There are 2,954 total COVID-19 cases, 54 total deaths, and 2,684 total recoveries in Thailand according to the reports on April $30^{\text {th }}, 2020$. The situation affects Thailand' economy and society in that all parts of Thailand have stuck for a while. However, Thailand's government has tried to solve this problem via administrative policies. 
The flattened curve policy is one of the policies without vaccination by trying to stop spreading coronavirus disease 2019 before the inflation rate of the coronavirus 2019 disease soared sharply and to protect people from the coronavirus 2019 particles. Here, the flattened curve policy in Thailand consists of social distancing by staying alone at home and working from home, physically distancing more than 2 $\mathrm{m}$ between 2 people or amongst people, wearing masks in communities, frequently washing hands with alcohol hand gel and soap, etc. The important point for the flattened curve in Thailand is the lockdown of the country by the emergency decree from 26 March to April $30^{\text {th }}, 2020$, and curfew law between 10 p.m. and 4 a.m. from April $3^{\text {rd }}$ to April $30^{\text {th }}, 2020$, for controlling the coronavirus 2019 disease spreading. Some examples of the details in the emergency decree include "People are not allowed to access the infectious and risk area, all the methods to enter Thailand are closed, people are not allowed to gather or assembly in a group, etc". Furthermore, Thailand's government may still extend the emergency decree and curfew law for another month. However, this research will be focused on March $26^{\text {th }}$ to April $30^{\text {th }}$, 2020 for the flattened curve policy.

The growth curve of infectious cases is one of the factors leading to the design of the government's policy around the world. It is a tool for monitoring the situation of severe infectious cases and controlling the waves of the number of patients. Empirical studies have been conducted on the relationship between real data and the estimate curves. The introduction of population growth curves was proposed with population size, the number of individuals in the population density, animal and human population growth, and the number of individuals in a particular area [1]. The exponential growth curve model was initiated to estimate the real data. The decrease in energy flux and increase in aerobic glycolysis were investigated by a constant growth rate of the exponential growth curve model [2]. The population of bacteria was assumed to the exponential growth curve model in biology [3]. The stage of the bacterial growth cycle which is the exponential growth was studied in the transcription process [4]. Moreover, the exponential growth curve model was applied to the financial field in the compound interest rate [5]. Then, the logistic growth curve model was developed to be an alternative to the exponential growth curve model. It was firstly proposed in studying autocatalysis and extended to animal science [6]. The relationship between poultry weight and time was described by the logistic growth curve of the time series [7]. To describe the growth of broiler, the logistic growth curve model was employed to predict broiler chicks [8]. The bodyweight of a commercial strain and chickens was measured and predicted by the logistic growth curve model [9]. The comparison between the exponential growth curve model and the logistic growth curve model was studied in environmental science based on the information from the ecology population [10].

In the fitting curve of the number of total COVID-19 cases, the parameters of the model were estimated. The least square method is one of the methods for estimating parameters. The estimation based on the least square method for estimating parameters of linear, power, exponential function was fit as interpolation and extrapolation of data [11]. The method for estimating parameters of the exponential growth and the logistic exponential growth curve models were studied in numerical method [12, 13]. Analysis and forecasting of the population which was represented by the logistic growth curve model of Bangladesh were evaluated by the least square method and Runge-Kutta method [14]. Furthermore, the least square error method was also applied to fit the Gompertz curve, and the necessary and sufficient conditions for the existence of the least square estimator were provided [15]. Moreover, the predictive models of the number of total COVID-19 cases for the herd immunity policy without vaccination in Sweden were presented. The herd immunity policy in Sweden was compared amongst the other Nordic countries (Norway, Finland, and Denmark) which were applied to the flattened curve policy [16].

Therefore, the estimation and forecasting of the number of total COVID-19 cases in Thailand are very important information for making decisions on Thailand's government and for announcing the administrative policies for controlling and protecting the outbreak of the COVID-19 diseases. The assumption for this research is to study the growth curve of the number of cumulative COVID-19 cases without vaccination for the flattened curve policy compared with the herd immunity policy. This research examines the suitable growth curve model and its estimated parameters by the least square method to describe the number of total COVID19 cases in Thailand. Also, the spreading rate of the number of the total COVID-19 cases in Thailand under the flattened curve policy was investigated. The comparison of parameters estimation between the exponential 
growth curve which represents the growth curve of the number of total COVID-19 cases in Thailand with the non-flattened curve policy and the logistic growth curve which represents the growth curve of the number of total COVID-19 cases in Thailand with the flattened curve policy is studied on the minimum of the root mean squared percentage error and the maximum of the coefficient of determination. The next section presents the mathematical and statistical background for the materials and methods of this research. In addition, the results, discussion, and conclusions are proposed for this research.

\section{Materials and methods}

This section presents the mathematical and statistical backgrounds of the research. The analysis was specified on the number of the cumulative COVID-19 cases without vaccination in Thailand on April $30^{\text {th }}, 2020$, which was the deadline date for the submission to the special issue on COVID-19 Pandemic Outbreak of Walailak Journal of Science and Technology (WJST). The number of the cumulative COVID-19 cases in Thailand as the data for this research was reported and was most updated on the midday of April $30^{\text {th }}, 2020$, on the Worldometer website [17].

This research assumes that the exponential growth curve model is for the number of the cumulative COVID-19 cases based on data from February $15^{\text {th }}$ to March $25^{\text {th }}, 2020$, without the flattened curve policy. The dates from February $15^{\text {th }}$ to March $25^{\text {th }}$, 2020 are the duration before the enactment of the emergency decree. The logistic growth curve model is for the number of cumulative COVID-19 cases based on the data from February $15^{\text {th }}$ to April $30^{\text {th }}, 2020$ with the flattened curve policy. The dates from February $15^{\text {th }}$ to April $30^{\text {th }}, 2020$ are the duration included in the emergency decree. Therefore, it is assumed that an exponential growth curve is developed for a non-flattened curve in which there is no policy for the fattening curve of the total COVID-19 cases. The total COVID-19 cases will likely exponentially increase for a non-flattened curve policy and the logistic growth curve is developed for the fattened curve in which the policy-the emergency decree- for controlling and protecting the COVID-19 outbreak is launched.

Thus, the growth curves of the population size model for estimating the number of cumulative COVID-19 cases in Thailand are designated as follows.

Let $C(t)$ be the population size of total COVID-19 cases in Thailand at time $t \geq 0$. The exponential growth curve model $[1,18]$ can be defined in an ordinary differential equation as;

$\frac{d}{d t} C(t)=r C(t) ; C(t=0)=C_{0}$

with initial population size $C_{0}>0$, growth rate parameter $r>0$.

The solution of Eq. (1) can be solved by a separable method as follows.

$d C(t)=r C(t) d t$

Taking integral into both sides of Eq. (1), it becomes;

$\int \frac{1}{C(t)} d C(t)=\int r d t$

$\ln |C(t)|=r t+A$

where $A$ is an arbitrary constant.

Taking exponential function into both sides, it becomes; 


$$
|C(t)|=\exp (r t+A)
$$

Thus, $C(t)=Z \exp (r t)$, where $Z= \pm \exp (-A)$

For initial time $(t=0), C_{o}=Z \exp (r \times 0)$

Thus, $Z=C_{0}$

Therefore, the solution of the exponential growth model is $C(t)=C_{0} \exp (r t)$.

The logistic growth curve model or logistic differential equation initially proposed by Verhulst [18] can be defined in ordinary differential equation as;

$$
\frac{d}{d t} C(t)=r C(t)\left[1-\frac{C(t)}{k}\right] ; C(t=0)=C_{0}
$$

with initial population size $C_{0}>0$, growth parameter $r>0$, carrying capacity parameter $k$.

The solution of Eq. (2) can be carried out as follows.

By separable method and taking the integral into both sides of Eq. (2), it becomes;

$$
\begin{aligned}
& \int \frac{1}{C(t)\left[1-\frac{C(t)}{k}\right]} d C(t)=\int r d t \\
& \int \frac{k}{C(t)[k-C(t)]} d C(t)=\int r d t
\end{aligned}
$$

By partial fraction, the term $\frac{k}{C(t)[k-C(t)]}=\frac{1}{C(t)}+\frac{1}{k-C(t)}$.

$$
\begin{aligned}
& \int\left(\frac{1}{C(t)}+\frac{1}{k-C(t)}\right) d C(t)=\int r d t \\
& \ln |C(t)|-\ln |k-C(t)|=r t+B \\
& \ln \left|\frac{C(t)}{k-C(t)}\right|=r t+B
\end{aligned}
$$

where $B$ is an arbitrary constant.

By taking exponential function into both sides of Eq. (3), it becomes;

$$
\left|\frac{C(t)}{k-C(t)}\right|=\exp (r t+B)
$$


$\frac{k-C(t)}{C(t)}=W \exp (-r t), \quad$ where $W= \pm \exp (-B)$

$k-C(t)=W C(t) \exp (-r t)$

$k=C(t)+W C(t) \exp (-r t)$

$k=C(t)[1+W \exp (-r t)]$

Thus, $C(t)=\frac{k}{[1+W \exp (-r t)]}$

For initial time $(t=0), \frac{k-C_{0}}{C_{o}}=W \exp (-r \times 0)$

Thus, $W=\frac{k-C_{0}}{C_{o}}$.

Therefore, the solution of logistic differential equation is $C(t)=\frac{k}{[1+W \exp (-r t)]}$ where $W=\frac{k-C_{0}}{C_{o}}$.

Next, when time tends to infinity $(t \rightarrow \infty)$, the $C(t)=C_{0} \exp (r t)$ of exponential growth model will tend to infinity and $C(t)=\frac{k}{[1+W \exp (-r t)]}$ of logistic growth model will tend to $k$ as;

$$
\begin{aligned}
C(t) & =\lim _{t \rightarrow \infty} \frac{k}{[1+W \exp (-r t)]} \\
& =k \lim _{t \rightarrow \infty} \frac{1}{[1+W \exp (-r t)]} \\
& =k
\end{aligned}
$$

Furthermore, the rate of growth of population size is $\frac{d}{d t} C(t)$. The maximum population size rate of growth which is the fastest growing population size for logistic growth model is $\frac{k}{2}$. The maximum of population size rate of growth will be carried out at the first derivatives with respect to $C(t)$ of $\frac{d}{d t} C(t)$ equaled to 0 as follows.

Let $f(C)=r C(t)\left[1-\frac{C(t)}{k}\right]=r C(t)-\frac{r}{k} C^{2}(t)$. 
$\frac{d}{d C(t)} f(C(t))=r-\frac{2 r}{k} C(t)=0$

Thus, $C(t)=\frac{k}{2}$.

Namely, the growth rate of population size reaches the peak point before starting to slow if the population size equals the carrying capacity parameter.

The least square estimation for parameters of the growth curve model is the method for estimation parameters with the minimum square of error for the model by fitting the observations $f(t)$ and analytical growth curves $C(t)$ which are derived above. Let $e(t)$ be the error between observations and analytical function; namely, $e\left(t_{i}\right)=f\left(t_{i}\right)-C\left(t_{i}\right) ; i=0,1,2, \ldots, n$ and $E$ be a sum of a square error function, i.e.

$$
E=\sum_{i=1}^{n}\left(e\left(t_{i}\right)\right)^{2}
$$

For the exponential growth curve function, $E\left(C_{0}, r\right)=\sum_{i=1}^{n}\left(C_{0} e^{r t_{i}}-f\left(t_{i}\right)\right)^{2}$. The minimum of $E$ will occur when $\frac{\partial E\left(C_{0}, r\right)}{\partial C_{0}}=0$ and $\frac{\partial E\left(C_{0}, r\right)}{\partial r}=0$. This results in the parameters $C_{0}$ and $r$ with the minimum sum of square error.

Likewise, for the logistic growth curve function, $\left.E(W, k, r)=\sum_{i=1}^{n} \frac{k}{\left[1+W \exp \left(-r t_{i}\right)\right]}-f\left(t_{i}\right)\right)^{2}$. The minimum of $E$ will occur when $\frac{\partial E(W, k, r)}{\partial W}=0, \frac{\partial E(W, k, r)}{\partial k}=0$ and $\frac{\partial E(W, k, r)}{\partial r}=0$. This results in the parameters $W, k$ and $r$ with the minimum sum of square error.

Estimation of the derivatives of the population size of total COVID-19 cases in Thailand is to study the change of the number of total COVID-19 over time changing. It depends on the limit, forward difference, and backward difference as follows. Thus,

$$
\begin{aligned}
& C^{\prime}(t)=\text { the population size growth rate of } C(t) \\
& =\lim _{h \rightarrow \infty} \frac{C(t+h)-C(t)}{h} \\
& =\lim _{\Delta t \rightarrow \infty} \frac{\Delta C(t)}{\Delta t} ; \Delta t=h \\
& \approx \frac{C_{B}(t)+C_{F}(t)}{2} \\
& \text { where } C_{B}(t)=\frac{C(t)-C(t-1)}{\Delta t}, C_{F}(t)=\frac{C(t+1)-C(t)}{\Delta t} .
\end{aligned}
$$


Thus, the estimated derivative which is represented by the spread of the number of total COVID-19 cases can be approximated by $\frac{C_{B}(t)+C_{F}(t)}{2}$.

To compare the efficiency of the model for estimation, the Root Mean Squared Percentage Error ( $R M S P E$ ) is used to compare the errors from the model for this research. The efficient model should be the minimum RMSPE. The RMSPE can be defined as;

$$
R M S P E=\sqrt{\frac{1}{n} \sum_{i=1}^{n}\left(\frac{C\left(t_{i}\right)-f\left(t_{i}\right)}{C\left(t_{i}\right)}\right)^{2}}
$$

where $C\left(t_{i}\right)$ is the population size from the growth curve model at time $t_{i}>0$,

$f\left(t_{i}\right)$ is the observation of the actual population size at time $t_{i}>0$.

Also, the coefficient of determination $\left(R^{2}\right)$ which represents the percentage of the growth curve model estimated by the least square method how much it is predicted by the independent variable time. In other words, the coefficient of determination is a ratio between the explained variance and the total variance.

By the formula in Eq. (5), $0 \leq R^{2} \leq 1$, if $R^{2}$ tends to 1 , it means that the growth curve model is almost predicted by the independent variable time.

$$
\begin{aligned}
R^{2} & =\frac{\text { Explained variance }}{\text { Total variance }} \\
R^{2} & =\frac{\sum_{i=1}^{n}\left(C\left(t_{i}\right)-\overline{f(t)}\right)^{2}}{\sum_{i=1}^{n}\left(f\left(t_{i}\right)-\overline{f(t)}\right)^{2}}
\end{aligned}
$$

where $\sum_{i=1}^{n}\left(C\left(t_{i}\right)-\overline{f(t)}\right)^{2}$ is the explained variance and $\sum_{i=1}^{n}\left(f\left(t_{i}\right)-\overline{f(t)}\right)^{2}$ is the total variance.

\section{Results and discussion}

In this section, the results from the method and the interpretation of the results are discussed. Observations or data which are the number of cumulative COVID-19 cases by day in Thailand from February $15^{\text {th }}, 2020$ to April $30^{\text {th }}, 2020$ are collected from the website [17]. The independent variables time in the growth curve models starts at the time corresponding to the day February $15^{\text {th }}, 2020$ to the time corresponding to the day April $30^{\text {th }}, 2020$. The period before the emergency decree defines as [Feb $15^{\text {th }}, 2020$ ] to [March $25^{\text {th }}, 2020$ ] and the period after the emergency decree [March 26 $6^{\text {th }}, 2020$ ] to [April $\left.30^{\text {th }}, 2020\right]$ which is a flattened curve policy. Also, fundamental statistics for the data are as follows. The interesting statistics in Table 1 are kurtosis and skewness because they represent the distribution of data. In other words, the valued of the kurtosis was $-1.69<3$, indicating that the distribution of data is platykurtic. The value of the skewness was 0.32 , which is between -0.50 and 0.50 , indicating that the distribution of data is symmetrical. However, the further study about this data should be focused on the determination of the probability density function of this data. It can indicate the expected value, variance, inference statistic to the population, confidence interval of the parameter, etc., if the probability density function of data is known. 
Table 1 Descriptive statistics for total COVID-19 cases by day in Thailand.

\begin{tabular}{cc}
\hline Statistics & Values \\
\hline Mean & 1199.04 \\
Standard Error & 136.45 \\
Median & 774.00 \\
Mode & 35.00 \\
Standard Deviation & 1189.58 \\
Sample Variance & 1415102.55 \\
Kurtosis & -1.69 \\
Skewness & 0.32 \\
Range & 2920.00 \\
Minimum & 34.00 \\
Maximum & 2954.00 \\
Sum & 91127.00 \\
Count & 76.00 \\
\hline
\end{tabular}

The parameters for the exponential growth curve were estimated by the least square method by using the number of total COVID-19 cases in Thailand from February 15 ${ }^{\text {th }}, 2020$ to March $25^{\text {th }}, 2020$ which is the period before the emergency decree announcement. The results showed that the parameters $C_{0}=16.07, r=0.08$. Thus, the estimated exponential growth curve for the number of total COVID-19 cases in Thailand is

$C(t)=16.07 \exp (0.08 t) ; t \geq 0$

For the accuracy of the estimated exponential growth curve model, $R M S P E=0.52$ and $R^{2}=0.68$.

Figure 1 shows the exponential growth curve model for estimating the number of total COVID-19 cases in Thailand. In the estimated exponential growth curve model, the number of total COVID-19 cases in Thailand initially grows slowly and steadily and it rapidly grows at time $t=34$. The estimated exponential growth curve model still is less than the actual number of total COVID-19 cases in Thailand for some time - about time $t=35$ to $t=68$. That may be a disadvantage of the exponential growth curve model. However, in the case of the non- flattened curve policy, the estimate exponential growth curve model will be overcome by the actual number of COVID-19 cases in Thailand in time $t=69$ and then it exponentially grows. It is realized that the actual number of total COVID-19 cases in Thailand has been flattened by comparing the estimated exponential growth curve after the emergency decree announcement because the estimated exponential growth curve grows sharply without the emergency decree announcement case. 


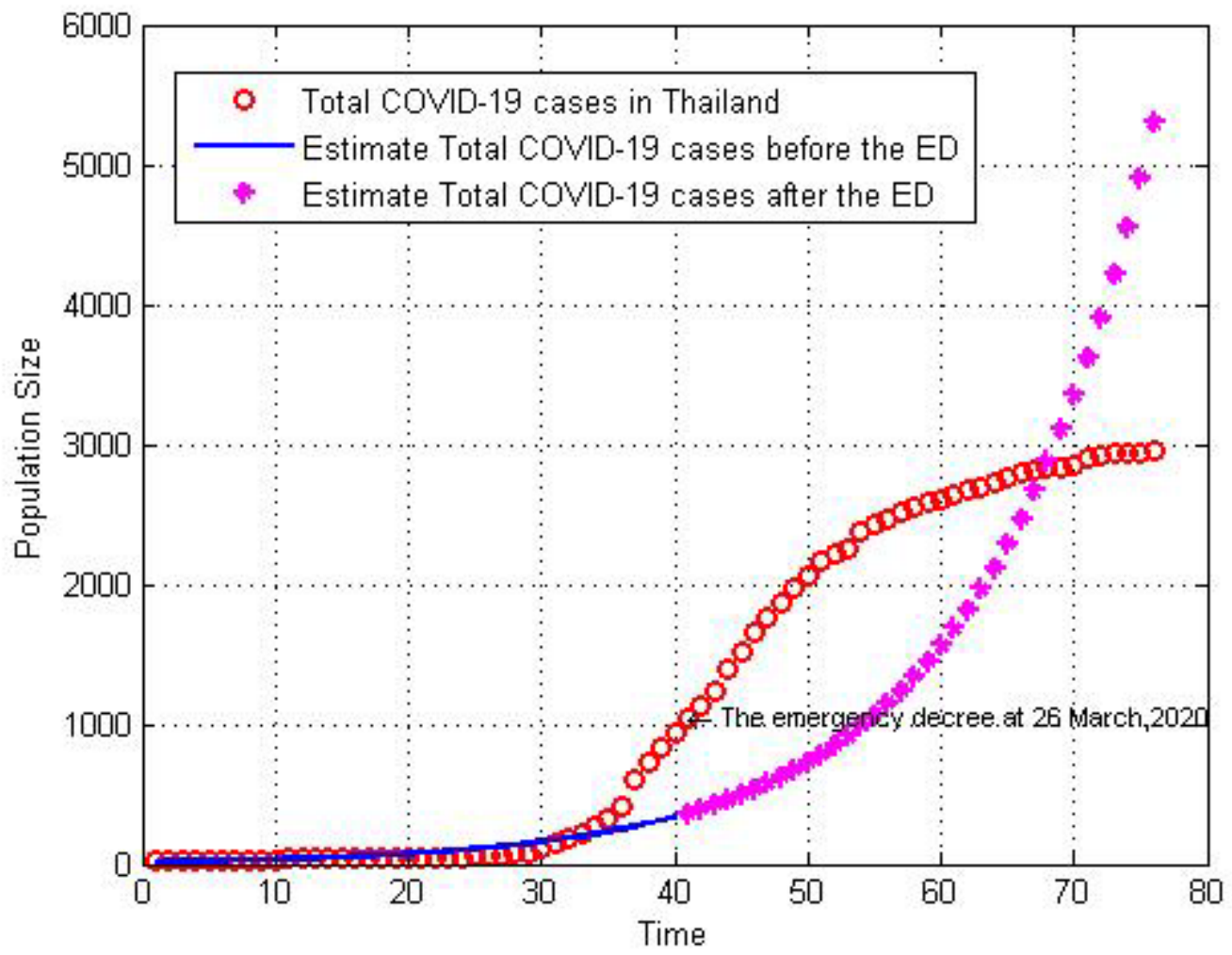

Figure 1 Estimated exponential growth curve before and after the Emergency Decree (ED).

The parameters for the logistic growth curve were estimated by the least square method by using the number of total COVID-19 cases in Thailand from February $15^{\text {th }}, 2020$ to April $30^{\text {th }}, 2020$ which is based on flattened curve policy, including the emergency decree. The results showed that the parameters $C_{0}=1.18, r=0.18, k=2,864.07$. Thus, the estimated logistic growth curve for the number of total COVID-19 cases in Thailand is;

$$
C(t)=\frac{2,864.07}{1+2,426.59 \exp (-0.18 t)} ; t \geq 0
$$

For the accuracy of the estimated logistic growth model, $R M S P E=0.47$ and $R^{2}=0.99$. 


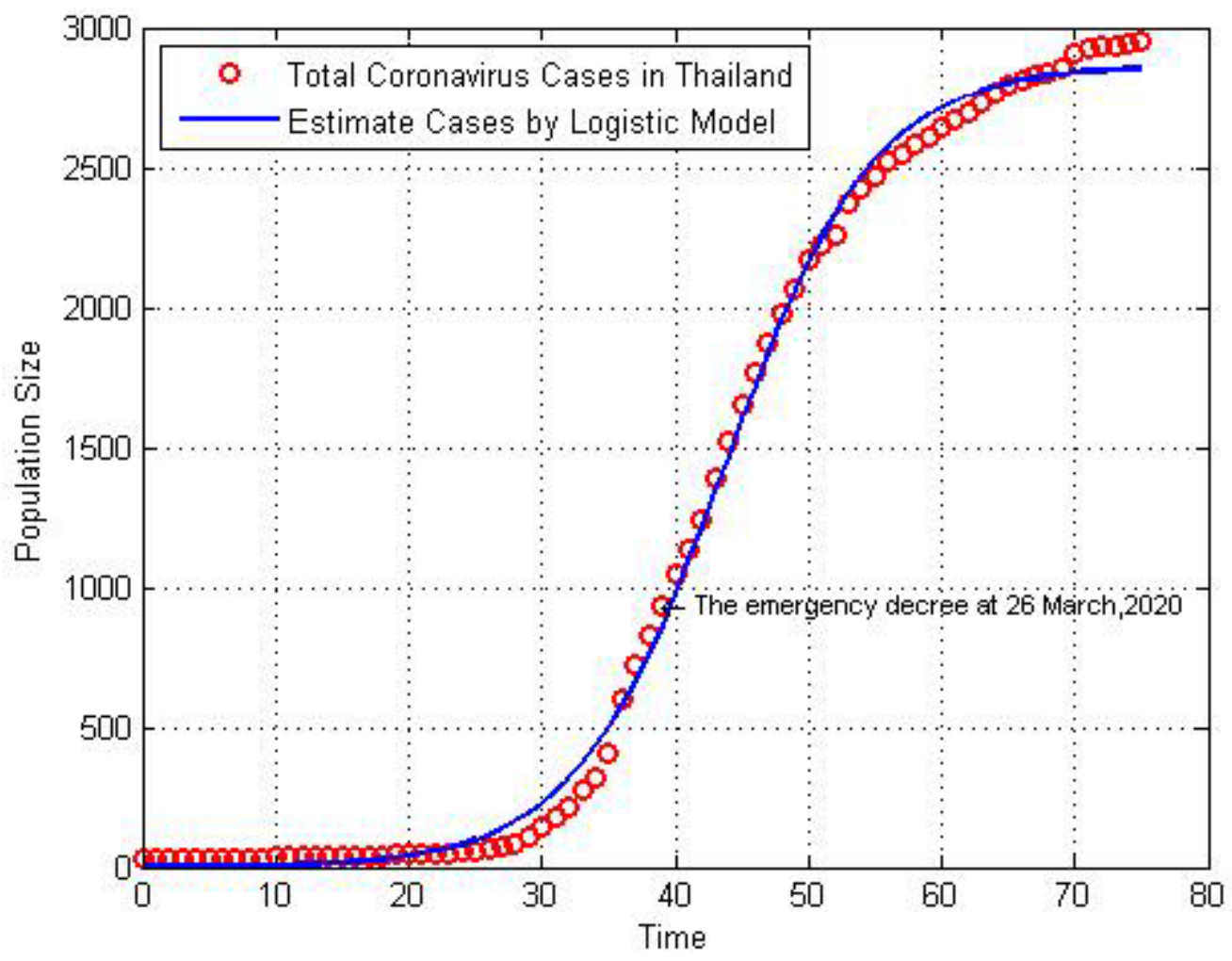

Figure 2 Estimated logistic growth curve.

Figure 2 illustrates the logistic growth curve for estimating the number of total COVID-19 cases in Thailand based on the flattened curve policy. The estimated logistic growth curve looks like the S-shaped curve. Based on the estimated logistic growth model, the number of total COVID-19 cases in Thailand will get the coronavirus disease 2019 is the carrying capacity 2,864.07 cases during the outbreak. The estimated logistic growth curve shows the fastest growth of the number of total COVID-19 cases in Thailand is approximately $1,432.03$ cases at time $t$ which can be solved by the equation;

$$
1,432.03=\frac{2,864.07}{1+2,426.59 \exp (-0.18 t)}
$$

Thus, the coronavirus disease 2019 will peak at $t=43.69 \approx 44$ days after the beginning day $(t=0$ ). The rate of growth of the number of total COVID-19 cases in Thailand is $C^{\prime}(44)$. The maximum value of rate of growth is at;

$$
\begin{aligned}
\frac{d}{d t} C(44)=0.18 C(44)\left[1-\frac{C(44)}{2,864.07}\right] & =0.18(1,432.03)\left[1-\frac{1,432.03}{2,864.07}\right] \\
& =127.74 \text { cases } / \text { day }
\end{aligned}
$$

Namely, at the peak of the outbreak $t \approx 44$, there are about 128 new people a day get the infection of the coronavirus disease 2019 in Thailand. The peak of the coronavirus disease 2019 in Thailand start to slow down at the $44^{\text {th }}$ day after the beginning day $(t=0)$. Furthermore, when $C^{*}\left(t_{*}\right)$ people had the 
coronavirus disease 2019 at any time $t_{*}$, the rate of spreading of the coronavirus disease 2019 in Thailand is $\frac{d}{d t} C\left(t_{*}\right)=0.18 C^{*}\left(t_{*}\right)\left[1-\frac{C^{*}\left(t_{*}\right)}{2,864.07}\right]$.

\section{Estimating the spread of COVID-19 Cases in Thailand}

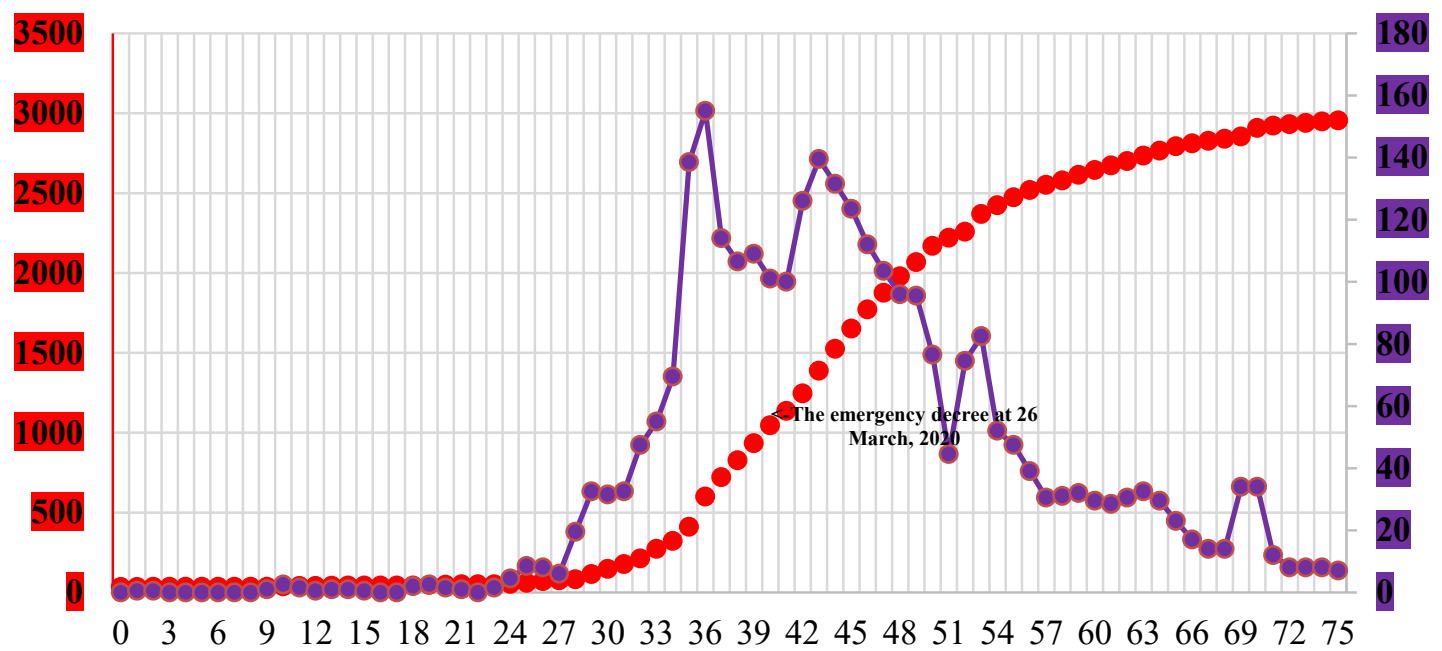

Figure 3 Estimate the derivative of the actual number of total COVID-19 cases.

Figure 3 demonstrates the growth rate which is represented by the derivative of the actual number of total COVID-19 cases in Thailand. The growth rate of the number of total COVID-19 infectious cases in Thailand can be classified into three periods. The first period about time $t=0$ to $t=27$, the speed of the number of COVID-19 cases in Thailand is fairly stable. The second period about $t=28$ to $t=36$, the speed of the number of COVID-19 cases in Thailand dramatically increases. The third period about time $t=37$ to $t=75$ it slumps quickly and slightly fluctuates inside the decreasing frame.

However, herd immunity policy is a policy based on unlocked country, no rules to control and protect COVID-19 spread, and no vaccination, etc. People under herd immunity can independently act in daily life without rules for fighting the COVID-19 particles. People can be infectious to COVID-19 to hope the herd immunity occurrence. Comparisons and examples between the flattened curve policy and the herd immunity policy are discussed. Sweden is the country in which the herd immunity policy has firstly been used to protect and control the COVID-10 outbreak since the worldwide pandemic time of coronavirus disease 2019 [16]. The other Nordic countries-Norway, Finland, and Denmark-including Thailand have applied the flattened curve policy to fight the COVID-19 outbreak. It is likely that Sweden has at least three waves of coronavirus disease 2019. The ratio of the number of the total COVID-19 deaths to the total COVID-19 cases is abbreviated as D/C and the ratio of the total COVID-19 deaths to 100,000 populations is abbreviated as $\mathrm{D} / \mathrm{P}$. The ratios $\mathrm{D} / \mathrm{C}$ and $\mathrm{D} / \mathrm{P}$ are shown as in Table 2. 
Table 2 Comparison COVID-19 outbreak between herd immunity and flattened curve policy.

\begin{tabular}{llllll}
\hline \multirow{2}{*}{ Variables } & \multicolumn{1}{c}{ Herd immunity policy } & \multicolumn{4}{c}{ Flattened curve policy } \\
\cline { 2 - 6 } & Sweden & Norway & Finland & Denmark & Thailand \\
\hline Populations & $10,146,474$ & $5,452,999$ & $5,547,184$ & $5,807,509$ & $69,931,468$ \\
Total COVID-19 deaths & 13,498 & 673 & 846 & 2,424 & 94 \\
Total COVID-19 cases & 813,191 & 96,770 & 78,672 & 231,973 & 28,947 \\
D/C ratio & $1.66 \%$ & $0.70 \%$ & $1.08 \%$ & $1.04 \%$ & $0.32 \%$ \\
D/P ratio & 133.03 & 12.34 & 15.25 & 41.74 & 0.13 \\
\hline
\end{tabular}

Table 2 shows the data [17] related to COVID-19 outbreak from February $15^{\text {th }}, 2020$ to April $2^{\text {nd }}$, 2021 amongst countries under herd immunity policy and flattened curve policy. The results showed that $\mathrm{D} / \mathrm{C}$ ratio and $\mathrm{D} / \mathrm{P}$ ratio of Sweden in which has applied the herd immunity policy to protect the COVID19 outbreak are higher than all of the other countries in which have applied the flattened curve policy. Especially, the $\mathrm{D} / \mathrm{C}$ ratio and $\mathrm{D} / \mathrm{P}$ ratio of Thailand which has applied the flattened curve policy are the lowest values when it is compared with the other countries. Therefore, Sweden should return to protect COVID-19 outbreak based on the flattened curve policy, such as the country locking down, social distancing, and mask wearing, self-quarantining, etc.

\section{Conclusions}

This research estimated the suitable growth curve for the number of the total COVID-19 cases in Thailand under the flattened curve policy and estimated the parameters of the suitable growth curves by the least square method. The findings revealed that the logistic growth curve model suited estimating the number of the total COVID-19 cases in Thailand with $R M S P E=0.47$ and $R^{2}=0.99$. The maximum number of the total infectious people in Thailand was approximately 2,864 cases based on the logistic growth curve model which was assumed under the flattened curve policy. However, the exponential growth curve model which was assumed without the flattened curve policy showed that the number of the total infectious people in Thailand soared steeply over the actual number in time $t \approx 68$ days after the beginning day $(t=0)$. Moreover, the maximum number of the epidemic based on the logistic growth curve model was in time $t \approx 44$ after the beginning day $(t=0)$ with about 128 new infectious people a day. In the approximation of the speed of COVID-19 spread in Thailand, the finding disclosed that it reached a peak point at time $t \approx 44$ days after the beginning day $(t=0)$, and then it declined sharply. Based on the number of the total COVID-19 cases in Thailand and the maximum value of the coefficient of determination between the logistic growth curve and the exponential growth curve, the logistic growth curve seems to provide a higher coefficient of determination than the exponential growth curve. Similarly, the minimum value of the root means squared percentage error between the logistic growth curve and the exponential growth curve was in the flavor of the logistic growth curve. Therefore, the logistic growth model based on flattened curve policy is an alternative model based on mathematical and statistical analysis and suitable for describing the number of the total COVID-19 cases in Thailand. On the whole, the speed of the number of total COVID-19 cases in Thailand is likely decreasing (analyzed on April $\left.30^{\text {th }}, 2020\right)$. However, there may still be residuals from the estimation of the logistic growth curve model and other factors to estimate the model. Compared with the herd immunity policy, the flattened curve policy should be selected to control and protect the COVID-19 spread. Further analysis should be focused on other growth curve models and other methods to analyze the number of total COVID-19 cases in Thailand. 


\section{References}

[1] SB Snider and JN Brimlow. An introduction to population growth. Nat. Educ. Knowl. 2013; 4, 3.

[2] S Nikolai, B Bogdan, S David, A Edoardo and V Alexander. Constant growth rate can be supported by decreasing energy flux and increasing aerobic glycolysis. Cell Rep. 2014; 7, 705-14.

[3] J Allen and B Waclaw. Bacterial growth: A statistical physicist's guide. Rep. Prog Phys. 2019; 82, $1-55$.

[4] MD Rolfe, CJ Rice, S Lucchini, C Pin, A Thompson, ADS Cameron, M Alston, MF Stringer, RP Betts, J Baranyi, MW Peck and JCD Hinton. Lag phase is a distinct growth phase that prepares bacteria for exponential growth and involves transient metal accumulation. J. Bacteriol. 2012; 194, 686-701.

[5] P Mariappan. Business mathematics, Pearson India Education Service, Delhi, 2015.

[6] TB Robertson. On the normal rate of growth of an individual and its biochemical significance. Archiv für Entwicklungsmechanik der Organismen. 1908; 25, 581-14.

[7] J France, J Dijkstra and MS Dhanoa. Growth functions and their application in animal science. Anim. Res. 1996; 45, 165-74.

[8] F Rashad and AS Firas. Growth curve of commercial broiler as predicted by different nonlinear functions. Am. J. Appl. Sci. Res. 2015; 1, 6-9.

[9] A Moharrery and M Mirzaei. Growth characteristics of commercial broiler and native chickens as predicted by different growth functions. J. Anim. Feed Sci. 2014; 23, 82-9.

[10] J Vandermeer. How populations grow: The exponential and logistic equations. Nat. Educ. Knowl. $2010 ; \mathbf{3}, 15$.

[11] JW Cain. Mathematics of fitting scientific data. In: RD Wells, JS Bond, J Klinman and BSS Masters (Eds.). Molecular Life Sciences. Springer Science+Business Media, New York, 2014, p. 668-73.

[12] A Kharab and RB Guenther. An introduction to numerical methods A MATLAB approach. CRC Press, Florida, 2012.

[13] D Jukić and R Scitovski. Solution of the least-squares problem for logistic function. J. Comput. Appl. Math. 2003; 156, 159-77.

[14] MS UllaH, G Mostafa, N Jahan and MAH Khan. Analyzing and projection of future Bangladesh population using logistic growth model. Int. J. Mod. Nonlinear Theor. Appl. 2019; 8, 53-61.

[15] J Dragan, K Gordana and S Rudolf. Least-squares fitting Gompertz curve. J. Comput. Appl. Math. 2004; 169, 359-75.

[16] R Sunthornwat and Y Areepong. Predictive model for the total daily COVID-19 cases with herd immunity policy. Chiang Mai Univ. J. Nat. Sci. 2021; 20, e2021016.

[17] Worldometer, Available at: https://www.worldometers.info/coronavirus/country/thailand, accessed April 2020.

[18] PF Verhulst. Notice sur la loi que la population suit dans son accroissement. Correspondence Mathematique et Physique (Ghent). 1838; 10, 113-21. 